\title{
Percepción de imagen corporal como factor condicionante del índice de masa corporal en médicos residentes del Hospital de Clínicas
}

\author{
Judith M. Torales ${ }^{1}$, Nelson J. Aquino ${ }^{1}$, Laura B. García ${ }^{1}$, Karina E. Scavenius ${ }^{1}$, Orlando R. Sequeira ${ }^{1}$, Luis

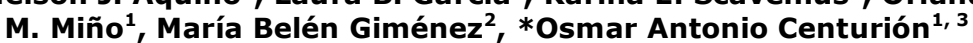 \\ ${ }^{1}$ División de Medicina Cardiovascular. Facultad de Ciencias Médicas-UNA. Hospital de Clínicas. San Lorenzo, \\ Paraguay \\ ${ }^{2}$ Cátedra de Medicina Familiar. Facultad de Ciencias Médicas-UNA. Hospital de Clínicas. San Lorenzo, Paraguay \\ ${ }^{3}$ Departamento de Investigación en Ciencias de la Salud. Sanatorio Metropolitano. Fernando de la Mora, Paraguay
}

Cómo referenciar este artículo/

How to reference this article:
Torales JM, Aquino NJ, García LB, Scavenius KE, Sequeira OR, Miño LM, Giménez MB, Centurión OA. Percepción de imagen corporal como factor condicionante del índice de masa corporal en médicos residentes del Hospital de Clínicas. Mem. Inst. Investig. Cienc. Salud. 2018; 16(2): 79-83

\section{RE S U M E N}

Uno de los factores condicionantes del peso corporal es la percepción que la persona tiene de su imagen corporal. El objetivo fue conocer la percepción de imagen corporal según el índice de masa corporal (IMC) en 107 Médicos Residentes del Hospital de Clínicas.Se utilizó un diseño observacional descriptivo de corte transverso. Se exploraron las variables edad, sexo, circunferencia abdominal, peso, talla, IMC, percepción del peso corporal. La circunferencia abdominal fue de riesgo elevado en $37 \%$ de los varones y en $16 \%$ de las mujeres, de riesgo muy elevado en $13 \%$ de varones y en $29 \%$ de las mujeres $(p=0,009)$. En cuanto al IMC la media fue de $25,7 \pm 4,6$; estando en peso normal el $45 \%$ y en sobrepeso el $43 \%$. Se encontró más mujeres con peso normal ( $63 \%$ vs $28 \%$ ). Los varones presentan más sobrepeso $(61 \%$ vs $28 \%)(p=0,0009)$. En cuanto a la percepción del peso corporal se perciben con obesidad 23\%; con sobrepeso $47 \%$ y con peso normal $28 \%$. Cruzando los datos con los IMC verdaderos, tenemos que el $43 \%$ no tiene la percepción real de acuerdo a su IMC. La concordancia en cuanto a IMC real y la percepción es del 63\%. Casi la mitad de los residentes estudiados no tiene la percepción real de acuerdo a su IMC. La percepción de obesidad es mayor en mujeres que en hombres acorde a su IMC real. Se requiere de más investigaciones en referencia al tema aplicándolo a toda la población del hospital para crear medidas educativas de oportuna intervención en la salud del personal sanitario.

Palabras clave: Percepción de imagen corporal, Médicos residentes, Índice de masa corporal.

\section{Perception of body image as conditioning factor of body mass index in medical residents at Clinical Hospital}

\begin{abstract}
A B S T R A C T
The perception of their own body image that a person has is a conditioning factor of the body weight. We aimed to know the perception of body image assessing the body mass index (BMI) in 107 medical residents of the Clinical Hospital. We utilized a descriptive observational design to evaluate multiple variables like gender, age, abdominal diameter, weight, height, body mass index, and perception of body weight. The abdominal diameter was of high risk in $37 \%$ of males and $16 \%$ in females, and of very high risk in $13 \%$ of males and in $29 \%$ of females $(p=0,009)$. The medium BMI was $25,7 \pm 4,6$. There was a normal BMI in $45 \%$ of the subjects and overweight in $43 \%$. There were more female with normal weight than male (63\% vs $28 \%)$, and there were more overweight male than female (61\% vs
\end{abstract}

Fecha de recepción: febrero 2018. Fecha de aceptación: marzo 2018

*Autor correspondiente: Prof. Dr. Osmar Antonio Centurión, MD, PhD, FACC, FAHA. Profesor Titular de Medicina. Facultad de Ciencias Médicas. Universidad Nacional de Asunción-UNA. Jefe de la División de Medicina Cardiovascular. Hospital de Clínicas. Dirección: Av. Mariscal López e/ Coronel Cazal. San Lorenzo, Paraguay. Teléfono: +(595) 971354444

Email: osmarcenturion@hotmail.com 
$28 \%)(p=0,0009)$. Considering the perception of body weight, $23 \%$ perceive themselves to be obese, $47 \%$ with overweight, and $28 \%$ with normal body weight. Comparing this perception with real BMI, we observed that $43 \%$ of the subjects do not have a real perception according to the BMI. The agreement between real BMI and the image perception is $63 \%$. Almost half of the medical residents studied do not have a real body weight perception according to BMI. The perception of obesity is greater in women than in men according to the real BMI. There is a necessity of larger studies including the total population of the hospital to create educational measures for timely intervention in the health of hospital personal.

Keywords: Body image perception; Medical residents; Body mass index.

\section{INTRODUCCIÓN}

El control de peso es un tema de interés sanitario prioritario debido al creciente número de personas que se enfrentan con problemas de sobrepeso/obesidad y por la importancia que tiene su padecimiento en el riesgo de sufrir diversas patologías como enfermedad cardiovascular, diabetes, e hipertensión arterial (1-4). Existe un elevado porcentaje de personas que padeciendo o no sobrepeso/obesidad, inician o mantienen dietas encaminadas a conseguir un adelgazamiento, más o menos importante.

Uno de los factores condicionantes del peso corporal es la percepción que la persona tiene de su imagen corporal. Diversos autores han destacado la baja concordancia que existe entre la imagen corporal y el estado nutricional (5-9). La imagen corporal condicionaría en parte las conductas alimentarias y la actitud o nivel de aceptación con relación a las dietas orientadas al control de peso (10-14). Por lo que conocer la percepción que la población tiene sobre su peso corporal nos ayudará a definir estrategias que permitan reforzar el concepto de peso normal o deseable, en caso de que los datos no muestren una adecuada concordancia.

El interés sobre estudios de percepción de la imagen corporal es cada vez mayor, por eso el objetivo de este estudio fue evaluar si existe concordancia entre la percepción corporal de los médicos residentes y su estado nutricional según el IMC, que además proporciona información valiosa sobre la importancia de intervenir tempranamente mediante una serie de estrategias educativas. De acuerdo a nuestro conocimiento no existe un trabajo similar en nuestro medio, motivo por el cual lo hace único en este contexto. Además, elegimos a esta población peculiar debido a que el personal de la salud es referencia de modelo a seguir del estilo de vida para los pacientes. Este hecho agrega al personal de blanco una carga adicional de responsabilidad a tener muy en cuenta en su vida profesional.

\section{MATERIALES Y MÉTODOS}

Estudio descriptivo, trasversal, realizado entre los meses de enero a marzo de 2016 y que incluyó a 107 residentes médicos en el Hospital de Clínicas, San Lorenzo, Paraguay y aceptaron completar el cuestionario. Fueron excluidos aquellos residentes con patologías de base. Los médicos residentes que aceptaron voluntariamente participar del cuestionario, previamente completando el consentimiento informado.

El proceso de recolección de datos se realizó con un cuestionario simple que contenían las siguientes variables: edad, sexo, circunferencia abdominal, peso, talla, índice de masa corporal y percepción del peso corporal.

Los datos fueron analizados con Microsoft Excel 2010 y Epi info 2007 CDC Atlanta, utilizándose estadística descriptiva y analítica. Para las asociaciones entre variables nominales se utilizó la prueba de chi cuadrado a un nivel de significancia de 0,05.

\section{RESULTADOS}

Se incluyeron 107 médicos residentes del Hospital de Clínicas, de los cuales el 52,3\% eran del sexo femenino y $47,7 \%$ del sexo masculino. La media de edad fue de $28,4 \pm 2,21$ (2438) años.

La circunferencia abdominal fue de riesgo elevado en $37,3 \%$ de los varones y $15,6 \%$ de las mujeres; de riesgo muy elevado en $12,5 \%$ de varones y en $28,6 \%$ de las mujeres $(p=0,009)$ como se observa en la Figura 1. 


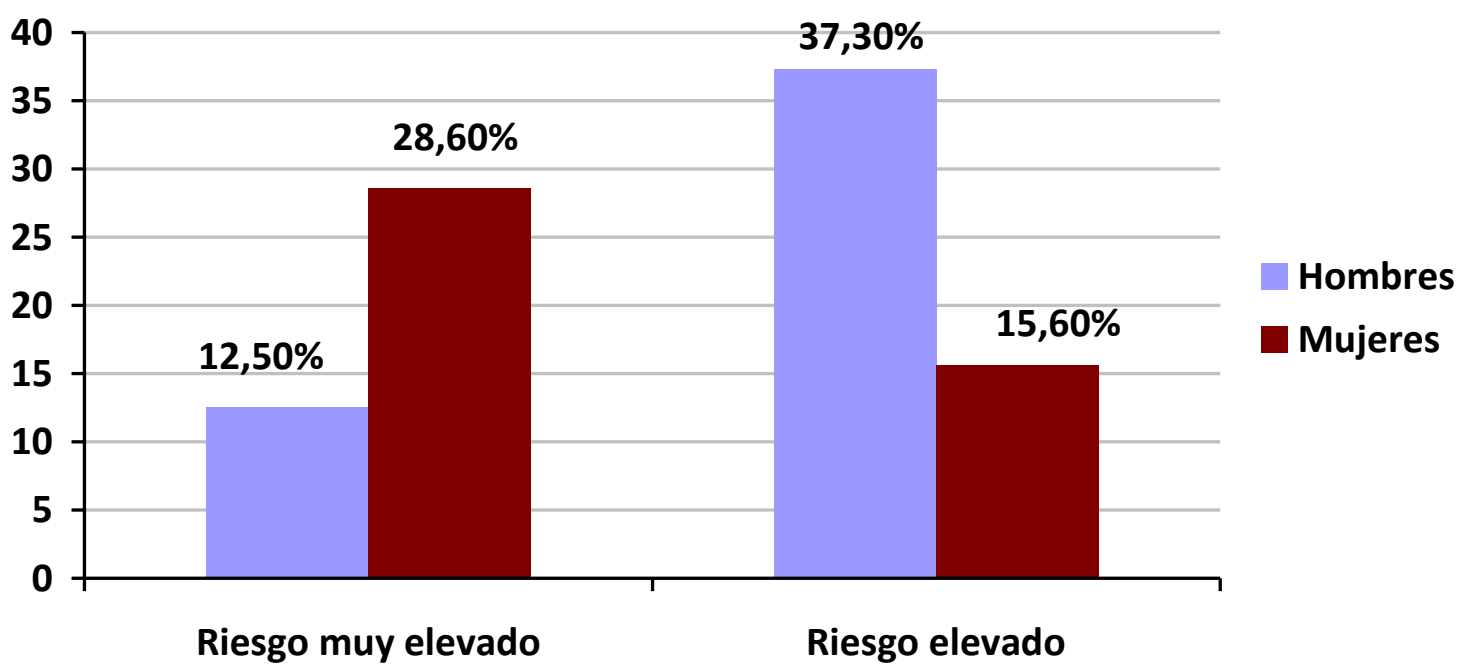

Figura 1. Incidencia del riesgo elevado y del riesgo muy elevado del parámetro de la circunferencia abdominal en ambos sexos.

En cuanto al IMC la media fue de $25,7 \pm 4,6(16,9-42)$; estando en peso normal el $44,8 \%$ y en sobrepeso $42,9 \%$; de los cuales un $5,6 \%$ presentaban obesidad Grado I; $2,8 \%$ presentaban obesidad Grado II y un 1,8\% presentaron obesidad Grado III (Tabla 1).

Tabla 1: Porcentaje correspondiente de acuerdo al índice de masa corporal

\begin{tabular}{lc}
\hline \multicolumn{1}{c}{ IMC } & Porcentaje (\%) \\
\hline Normopeso & 44,8 \\
Soprepeso & 42,9 \\
Obesidad G I & 5,6 \\
Obesidad G II & 2,8 \\
Obesidad G III & 1,8 \\
\hline
\end{tabular}

Realizando la comparación por sexo se encontraron más mujeres con peso normal que los varones $(62,9 \%$ vs $27,5 \%)$, en cambio los varones presentaron más sobrepeso que las mujeres $(60,8 \%$ vs $27,7 \%)$ siendo la diferencia estadísticamente significativa $(p=0,0009)$. En cuanto a la percepción del peso corporal, se perciben con obesidad el $23,4 \%$; con sobrepeso el $46,7 \%$ y con peso normal el $28,1 \%$ (Figura 2 ). 


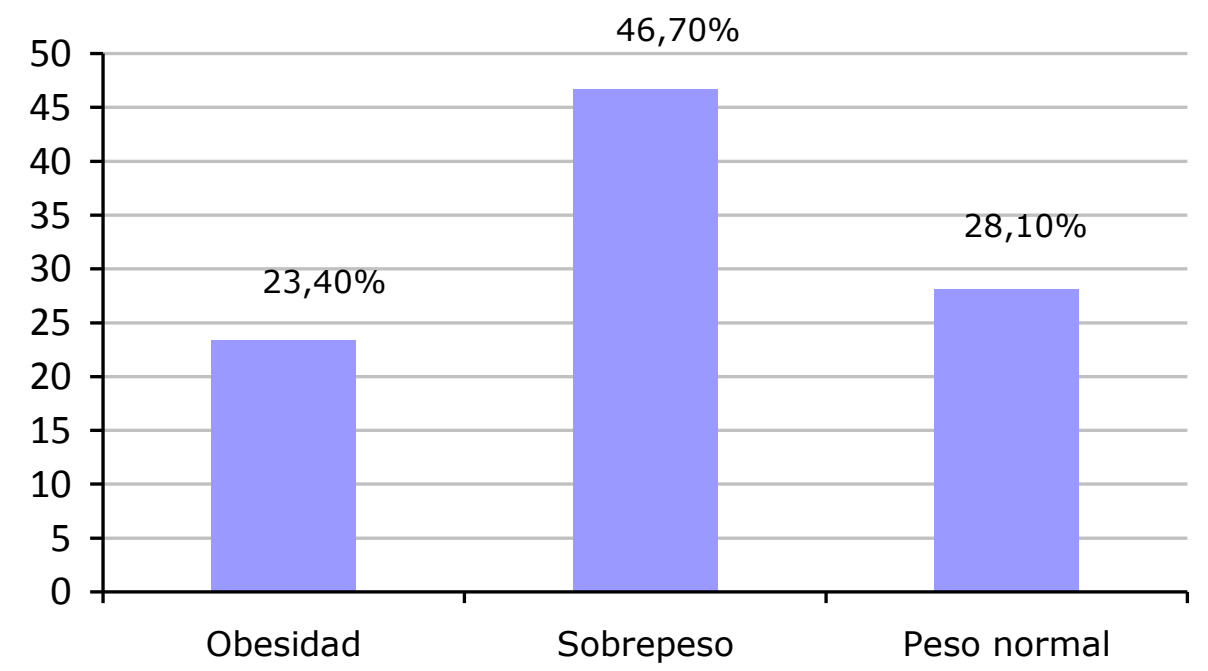

Figura 2. Percepción del peso corporal según el peso normal, obesidad, y el sobrepeso en los 107 residentes.

Cruzando los datos con los IMC verdaderos, tenemos que $43 \%$ no tiene la percepción real de acuerdo a su IMC. Siendo que $13(12,1 \%)$ se perciben como obesos no siendo obesos, $4(3,8 \%)$ se perciben con sobrepeso no teniendo sobrepeso y a la inversa 17 $(15,8 \%)$ que están en peso normal, no se perciben como tal. La concordancia en cuanto a IMC real y percepción es del 63\% (67 residentes).

\section{DISCUSIón}

En esta investigación realizada hemos constatado que el $43 \%$ de los residentes estudiados no tiene la percepción real de acuerdo a su IMC, esto es similar a el trabajo realizado por Ozuna y cols, aunque en dicho trabajo el porcentaje es menor, la sobrestimación del IMC fue de $24.5 \%$; en el trabajo realizado por Alcaraz y cols la sobreestimación de la imagen corporal fue del 68,4\%. La edad promedio de la población era de 28 años aproximadamente, similar al trabajo realizado por Alcaraz y cols en la cual la edad promedio era de 23 años. No es de extrañar que la percepción de obesidad sea mayor en mujeres que en hombres acorde a su IMC real $(9,10)$. Es de suma importancia realizar más investigaciones en referencia al tema aplicándolo a toda la población del hospital para crear medidas educativas de oportuna intervención en la salud del personal sanitario. Es conocido el hecho que existe un elevado número de personas que, ya sea por estética o por salud, emprenden medidas para perder peso corporal (11-13). Por lo tanto, es necesario adoptar las medidas adecuadas para evitar este tipo de prácticas y mejorar los conocimientos de la población en materia de nutrición. Estas decisiones de perder peso son frecuentemente poco adecuadas debido al deseo de adelgazar de una forma rápida y sin esfuerzo y a la falta de conocimientos sobre cuál es la dieta más adecuada para perder peso.

De acuerdo con los criterios establecidos para agrupar a los individuos según el IMC, el peso del colectivo estudiado se encuentra dentro de una situación de normalidad (1). El porcentaje de obesidad real de la población de estudio fue de $23,4 \%$, esto concuerda con las estadísticas nacionales donde se encontró una prevalencia de obesidad de $23,9 \%(2,14)$. A pesar de que un elevado porcentaje del colectivo estudiado presenta un peso considerado como normal, un $27,5 \%$ de los varones y un $62,9 \%$ de las mujeres $(p<0,001)$ consideran que su peso se encuentra por encima del peso más beneficioso para su salud y del peso considerado como más atractivo, por lo que se constata mayor grado de insatisfacción por la imagen corporal en las mujeres, esto concuerda con el trabajo realizado por CasillasEstrella y cols. Destaca el hecho de que el grado de insatisfacción es mayor entre las mujeres con peso normal, cuyo deseo por perder peso es mayor que entre las que realmente lo necesitan por presentar sobrepeso/obesidad. En algunos estudios realizados en mujeres también se ha encontrado esta insatisfacción y el deseo de perder peso aunque no se necesite $(9,10)$, lo que puede ser debido a que el colectivo femenino recibe desde edades tempranas constantes mensajes sobre los patrones de belleza y su relación con el 
éxito, personal y profesional. Si bien nuestra población estudiada tiene un número relativamente pequeño, lo cual podría ser una limitación de este estudio, los resultados obtenidos son muy similares a otras investigaciones con mayor número de pacientes.

Debido al elevado número de personas que, ya sea por estética o por salud, emprenden medidas para perder peso corporal es necesario adoptar las medidas adecuadas para evitar este tipo de prácticas y mejorar los conocimientos de la población en materia de nutrición. Estas decisiones de perder peso son frecuentemente poco adecuadas debido al deseo de adelgazar de una forma rápida y sin esfuerzo y a la falta de conocimientos sobre cuál es la dieta más adecuada para perder peso.

Se requiere de más investigaciones en referencia al tema aplicándolo a toda la población del hospital para crear medidas educativas de oportuna intervención en la salud del personal sanitario.

\section{REFERENCIAS BIBLIOGRÁFICAS}

1. D'Agostino RB Sr, Vasan RS, Pencina MJ, Wolf PA, Cobain M, Massaro JM, et al. General cardiovascularrisk profile for use in primary care: The Framingham Heart Study. Circulation, 2008; 117: 743-53.

2. Ministerio de Salud Pública y Bienestar Social. Primera Encuesta Nacional de Factores de Riesgo de Enfermedades No Transmisibles en población general. Asunción. Paraguay 2012.

3. World Health Organization. Obesity: Preventing and managing global epidemic. Geneva: WHO, 2014.

4. Manzur F, Alvear C, Alayón A. El perfil epidemiológico del sobrepeso y la obesidad y sus principales comorbilidades en la ciudad de Cartagena de Indias. Rev. Colom. Cardiol. 2009; 16(5): 194-200.

5. Osuna-Ramírez I, Hernández-Prado B, Campuzano JC, Salmerón J. Índice de masa corporal y percepción de la imagen corporal en una población adulta mexicana: la precisión del autorreporte. Salud Pública Méx (revista en internet). 2006 (citado 2017 Jul 31); 48(2): 94-103. Disponible en: http://www.scielo.org.mx/scielo.php?script=s ci_arttext\&pid=S0036-

36342006000200003\&Ing=es

6. Vaquero-Cristóbal $R$, Alacid F, Muyor JM, López-Miñarro PA. Body image: liteature review. Nutr. Hosp. 2013; 28(1):27-35. Disponible en:

http://scielo.iscii.es/scielo/.php?script=sci_art tex\&pid=S0212-1611201300010004\&Ing=es. http://dx.doi.org/10.3305/nh.2013.28.1.6016

7. Hidalgo-Rasmussen CA, Hidalgo-San Martin A. Percepción del peso corporal, comportamiento de control de peso y calidad de vida en adolescentes mexicanos estudiantes de secundaria. Rev. Mex. De trastor. Aliment 2011;2(2):71-81.
Disponible:http://www.scielo.org.mx/scielo. php?script $=$ sci_arttex $\&$ pid $=$ s200715232011000200003\&Ing =es

8. Alcaraz-López G, Lora-Gómez E, BerrioEstrada ME. Índice de masa corporal y percepción de la imagen corporal en estudiantes de enfermería. Index Enferm 2011; 20 (1-2): 11-5. Disponible en: 10.4321/S1132-12962011000100003.

9. Rodríguez-Guzmán L, Carballo-Gallegos $L$, Falcón-Coria A, Arias-Flores R, Puig-Nolasco Á. Autopercepción de la imagen corporal en mujeres y su correlación con el índice de masa corporal. Medicina Universitaria, Elsevier, Méx. 2010; 12(46): 29-32. Doi: 10.3305/nh.2011.26.3.4604

10. Casillas-Estrella M, Montaño-Castrejón N, Reyes-Velázquez $V$, Bacardí-Gascón $M$, Jiménez-Cruz A. A mayor IMC mayor grado de insatisfacción de la imagen corporal. Rev Biomed 2006;17:243-9.

11. Rodríguez-Guzmán L, Rodríguez-García R. Percepción de imagen corporal, índice de masa corporal y sobrepeso en estudiantes universitarios del Sureste. Rev Med Pediatr $2001 ; 68$ (4); 135-8.

12. Morán-Álvarez I, Cruz-Licea V, IñárrituPérez MC. El índice de masa corporal y la imagen corporal percibida como indicadores del estado nutricional en universitarios. Rev Fac Med UNAM 2007; 50(2): 76-9.

13. Sánchez-Villegas $A$, Madrigal $H$, MartínezGonzález MA, Kearney J, Gibney MJ, de Irala J, Martínez JA. Perception of body image as indicator of weight status in the European Union. J Hum Nutr Dietet 2001; 14:93-102.

14. Cañete F, Fretes G, Sequera VG, Tumes C, Santacruz E, Paiva T et al. Epidemiología de la obesidad en el Paraguay An. Fac. Cienc. Méd. (Asunción) 2016;49(2):17-26. 